\title{
Acceso al agua potable Indicador clave de desarrollo humano
}

DOI: 10.22403/UQROOMX/TYP02/10

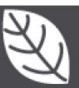

\author{
Oscar Frausto* \\ Thomas Ihl, Justo Rojas**
}

RESUMEN

El Observatorio Urbano de Cozumel ha señalado el primer camino a cumplir en la meta de reducir a la mitad, en el año 2015 , el porcentaje de personas que no cuenta con acceso sostenible a fuentes seguras de agua potable para garantizar la sostenibilidad del medio ambiente. Se reconoce que 2573 personas carecían de acceso al vital líquido en el año 2000, por lo que reducir a I 287 habitantes es la meta deseada en 20 I5. Conjuntamente, se reconocen las áreas de la ciudad con mayor déficit en el acceso del agua con el fin de impactar en eficiencia y eficacia las políticas públicas a desarrollar en un futuro.

Palabras Cozumel, agua, indicadores, objetivos del desarrollo del CLAVE $\mid$ milenio.

* Universidad de Quintana Roo, Unidad Cozumel, Cuerpo Académico de Turismo / ofrausto@uqroo.mx

**Observatorio Urbano de la Riviera Maya I

http:cozumel.uqroo.mx/cozumel/oulrm/index.htm 


\section{Introducción}

I72 Los Objetivos de Desarrollo del Milenio (ODM) representan un compromiso en la lucha contra la pobreza y buscan resolver los problemas que afectan al desarrollo humano (Unceta, 200I). Los ODM establecidos por la comunidad internacional en el año 2000 comprenden ocho objetivos, 18 metas específicas y 48 indicadores. Los ocho objetivos se relacionan con el tema del agua. Una de las metas específicas de los ODM, en términos numéricos y de tiempo, es "reducir a la mitad, en el año 2015, el porcentaje de personas que no cuenta con acceso sostenible a fuentes seguras de agua potable" (Guedán, 2005).

\section{Objetivos de Desarrollo del Milenio para 20 I5}

Los ocho ODM se describen a continuación, se observa el vínculo entre cada uno de ellos. El logro de un objetivo genera un efecto dominó ante los restantes siete, sin embargo, sin uno es imposible el desarrollo a largo plazo.

I. Reducir a la mitad la pobreza extrema y el hambre

Mil doscientos millones de personas viven con menos de un dólar por día. Sin embargo, 43 países, cuya población representa $60 \%$ de la población mundial, ya han alcanzado o están bien encaminados para lograr el objetivo de reducir a la mitad, para el año 2015, el porcentaje de personas que padecen hambre.

2. Reducir en tres cuartas partes la mortalidad materna

En el mundo en desarrollo, el riesgo de que la madre muera en el parto es uno por 48. Pero ahora casi todos los países tienen programas de maternidad segura y están listos para hacer progresos.

3. Lograr la enseñanza primaria universal

Ciento trece millones de niños no van a la escuela, pero el objetivo es alcanzable; por ejemplo, se prevé que $95 \%$ de los niños de la India asistirán a la escuela en 2015 . 
4. Reducir la propagación de enfermedades, especialmente el VIH/sida y el paludismo

Las enfermedades mortales han destruido una generación de logros del desarrollo. Países como el Brasil, Senegal,Tailandia y Uganda han demostrado que podemos detener la propagación del VIH.

5. Potenciar el papel de la mujer y promover la igualdad entre el hombre y la mujer

Dos tercios de los analfabetos del mundo son mujeres, y $80 \%$ de los refugiados son mujeres y niños. Desde la Cumbre sobre el Microcrédito celebrada en 1997, se han hecho progresos en la tarea de beneficiar y potenciar un número cada vez mayor de mujeres pobres (19 millones en 2000 solamente).

6. Reducir en dos terceras partes la mortalidad de los niños menores de cinco años

Cada año mueren II millones de niños pequeños, pero este número representa una disminución con respecto a los 15 millones de 1980.

7. Crear una asociación mundial para el desarrollo, con objetivos en materia de asistencia, comercio y alivio de la deuda

Demasiados países en desarrollo gastan más en el servicio de la deuda que en servicios sociales. Sin embargo, con la nueva asistencia prometida en la primera mitad de 2002 solamente, se llegará a 12000 millones de dólares adicionales por año en 2006.

8. Garantizar la sostenibilidad del medio ambiente

Más de I 000 millones de personas aún no tienen agua potable; sin embargo, en el decenio de 1990, casi I 000 millones de personas ganaron acceso al agua potable y otras tantas al saneamiento (Unceta, 200I).

\section{Monitoreo del agua para el logro de los ODM}

Monitorear el progreso de los ODM en el sector de agua es importante si se desea llevar adelante el compromiso político de la comunidad internacional y de los gobiernos nacionales. La información relacionada con el monitoreo es 
esencial para promover el tema del agua en las políticas y estrategias nacionales de reducción de la pobreza.

Se están desarrollando varias iniciativas relacionadas con el logro de los ODM; sin embargo, la información básica sobre el sector de agua sigue siendo insatisfactoria y se cuestiona la confiabilidad de los datos estadísticos. No se ha llegado a un consenso general con respecto a los instrumentos, metodologías y definiciones que deben utilizarse para el monitoreo de los ODM a nivel global, nacional y local y, al parecer, todavía no se ha establecido un sistema unificado y estandarizado.

El Objetivo Garantizar la sostenibilidad del medio ambiente tiene tres metas: a) Incorporar los principios del desarrollo sostenible en las políticas y los programas nacionales y revertir la pérdida de recursos del medio ambiente; b) Mejorar sustancialmente, para el año 2020, la vida de por lo menos 100 millones de habitantes de asentamientos precarios $y, c$ ) Reducir a la mitad el porcentaje de personas que carezcan de acceso sostenible al agua potable. Esta última es el objeto de discusión del presente escrito.

Ante esta problemática y para lograr las metas y el objetivo del milenio, la Secretaría de Desarrollo Social en México funda el programa Red Nacional de Observatorios Urbanos en conjunto con la ONU-Hábitat México. La primera coordina la implementación de Observatorios Urbanos Locales (OUL); la segunda, se encarga de reconocerlos a nivel internacional con el fin de presentar el registro y avance de los indicadores que conlleven a lograr los ODM.

La Red Nacional de Observatorios urbanos se constituye de 37 oul, tres de ellos en el estado de Quintana Roo: Cancún, Cozumel y Playa del Carmen, estos últimos se denominan Riviera Maya. Se ha dado un paso importante al desarrollar los 42 indicadores de Desarrollo Humano en los 37 oul, sin embargo resalta la tarea inmediata de revisar aquellos que influyen y determinan el avance de la reducción de la pobreza extrema y a la dotación de los servicios.

\section{Acceso al agua potable: indicador clave de desarrollo humano}

El acceso al agua potable es un indicador clave que nos permitirá asegurar la sustentabilidad ambiental y lograr la promoción de acceso a los servicios básicos. Se define como la "proporción de la población que usa cualquiera de los siguientes formas de suministro de agua para beber: agua entubada, toma 
pública, pozos protegidos, corrientes protegidas o agua de lluvia" (Sedesol, 2004). Su importancia radica en que el acceso al agua segura es una de las necesidades más apremiantes de los seres humanos. El suministro adecuado en cantidad y calidad es indispensable para garantizar su salud y su supervivencia. La población que carece de ella es segregada irremediablemente, pues tal condición es suficiente para que en muchos casos le sean negados sus derechos básicos.

El indicador se construye con base en información del XII Censo General de Población y Vivienda 2000 y el cálculo corresponde a:

Variables

OVPAP Ocupantes en viviendas particulares que no disponen de agua entubada y usan agua de pipa.

TOVP Total de ocupantes en viviendas particulares.

NE Ocupantes en viviendas particulares que no especificaron si disponían o no de agua potable.

KI4 Indicador de acceso al agua potable

Fórmula

$$
\mathrm{KI} 4=\frac{[\mathrm{I}-\text { OVPAP }]}{\text { TOVP }-\mathrm{NE}} \quad \mathrm{K} I 4=\frac{[\mathrm{I}-2573]}{58438.6-0}=95.60 \%
$$

\section{Resultados}

El indicador se ha señalado a nivel de AGEB (área geoestadística básica) de la ciudad de Cozumel, ésta se diferencia en 36 áreas (tabla I). En resumen, se tiene que 2573 habitantes carecen del acceso a agua potable. En las áreas periféricas de la ciudad, la carencia llega a alcanzar 30\% (colonias Juan Bautista Vega у стм) con 258 habitantes. Asimismo, con $20 \%$ de población sin acceso, se identifica a las colonias San Miguel I y II, con 692 habitantes. Finalmente, con $10 \%$ de carencia de agua entubada están registradas las colonias Centro, 10 de Abril, Emiliano Zapata, Adolfo López Mateos, Repobladores y San Gervasio, entre otras, sumando en total I 623 habitantes (véase mapa I). 
Acceso al agua potable Indicador clave

de desarrollo humano

Tabla I. Indicador deAcceso a agua potable por ageb de CozUmel

\begin{tabular}{|c|c|c|c|c|}
\hline AGEB & OVPAP & TOVP & NE & KI4 (\%) \\
\hline $002-8$ & 137 & 3256.24 & 0 & 95.78 \\
\hline $003-2$ & 246 & 3955.16 & 0 & 93.77 \\
\hline $005-1$ & 20 & 4488.44 & 0 & 99.55 \\
\hline $007-0$ & 97 & 1329.16 & 0 & 92.71 \\
\hline $040-3$ & 0 & 327.24 & 0 & 100.00 \\
\hline $04 \mid-8$ & 0 & 690.84 & 0 & 100.00 \\
\hline $042-2$ & 53 & 2254.32 & 0 & 97.67 \\
\hline 044-I & 485 & 4419.76 & 0 & 89.03 \\
\hline $045-6$ & 16 & 1106.96 & 0 & 98.54 \\
\hline $072-3$ & 234 & 1975.56 & 0 & 88.14 \\
\hline $073-8$ & 109 & 3159.28 & 0 & 96.55 \\
\hline $074-2$ & 16 & 601.96 & 0 & 97.32 \\
\hline $075-7$ & 36 & 941.32 & 0 & 96.14 \\
\hline $078-0$ & $14 \mid$ & 6019.6 & 0 & 97.65 \\
\hline $079-5$ & 65 & 2415.92 & 0 & 97.32 \\
\hline $080-8$ & 24 & 2767.4 & 0 & 99.12 \\
\hline $08 I-2$ & 61 & 2165.44 & 0 & 97.20 \\
\hline $082-7$ & 57 & 2565.4 & 0 & 97.80 \\
\hline $083-1$ & 194 & 2989.6 & 0 & 93.51 \\
\hline $089-9$ & 162 & 2775.48 & 0 & 94.18 \\
\hline 090-I & 53 & 1050.4 & 0 & 95.00 \\
\hline $091-6$ & 0 & 925.16 & 0 & 100.00 \\
\hline $092-0$ & 0 & 331.28 & 0 & 100.00 \\
\hline 094-A & 0 & 52.52 & 0 & 100.00 \\
\hline $097-3$ & 0 & 0 & 0 & 0.00 \\
\hline $098-8$ & 0 & 0 & 0 & 0.00 \\
\hline $099-2$ & 0 & 32.32 & 0 & 100.00 \\
\hline $100-7$ & 0 & 16.16 & 0 & 100.00 \\
\hline $101-1$ & 12 & 202 & 0 & 94.00 \\
\hline $102-6$ & 32 & 1292.8 & 0 & 97.50 \\
\hline $103-0$ & 53 & 2072.52 & 0 & 97.47 \\
\hline $104-5$ & 214 & 904.96 & 0 & 76.34 \\
\hline $105-A$ & 0 & 1001.92 & 0 & 100.00 \\
\hline $106-4$ & 0 & 0 & 0 & 0.00 \\
\hline $107-9$ & 0 & 153.52 & 0 & 100.00 \\
\hline $108-3$ & 44 & 189.88 & 0 & 76.60 \\
\hline Total Cozumel & 2573 & 58438.6 & 0 & 95.60 \\
\hline
\end{tabular}

Fuente: XII Censo General de Población y Vivienda 2000. Resultados definitivos. SCINCE 95. y Conteo de Población y Vivienda 1995. Resultados definitivos. SCINCE 95. 


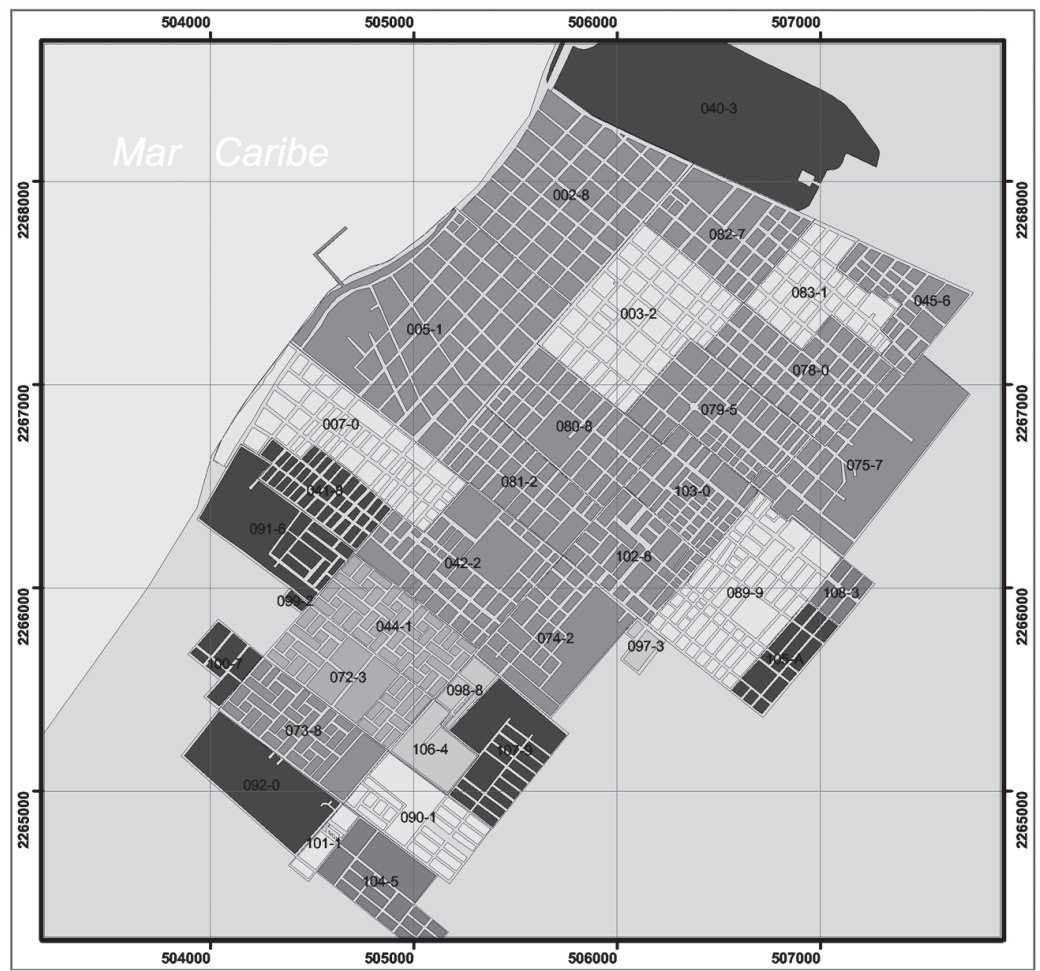

Mapa 1. Indicador Clave 4. Acceso a agua potable

Porcentaje de hogares con acceso a un suministro mejorado de agua.

Acceso a agua potable en $\%$

\begin{tabular}{l|l}
\hline & no data \\
& $70.00-80.00$ \\
\hline & $80.01-90.00$ \\
\hline & $90.01-95.00$ \\
\hline & $95.01-99.99$ \\
100.00
\end{tabular}
AGEB

Municipio Cozumel

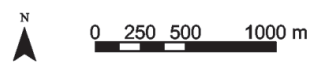

Proyección: NAD 1927 UTM Zone $16 \mathrm{~N}$

Fuente: INEGI 2000 y SEDESOL 2000

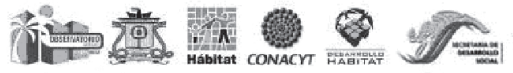


Así, la meta del milenio sobre la atención a acceso de agua potable en Cozumel (y se compara con Playa del Carmen) se observa a continuación:

El objetivo 7 de los ODM es el de garantizar la sostenibilidad del medio ambiente.

\begin{tabular}{lcc}
\hline \multicolumn{1}{c}{$\begin{array}{c}\text { Meta } \\
\begin{array}{l}\text { Reducir a la mitad el porcentaje } \\
\text { de personas que carezcan de } \\
\text { acceso sostenible al agua potable }\end{array}\end{array}$} & $\begin{array}{c}\text { Año } 2000 \\
\text { Habitantes (\% de la } \\
\text { población con acceso) }\end{array}$ & $\begin{array}{c}\text { Año 2015 } \\
\text { Habitantes (\% de la } \\
\text { población con acceso) }\end{array}$ \\
\hline Cozumel & $2573(95.6)$ & 1287 \\
Playa del Carmen & 3 II6 (92.6I) & 1558 \\
\hline
\end{tabular}

\section{Recomendaciones}

Los logros alcanzados en el monitoreo de los ODM a nivel local en materia de agua, y los desafíos existentes al 2015, han permitido la formulación de varias recomendaciones que toman en cuenta la interdependencia de los diferentes desafíos y sus posibles soluciones. Estas recomendaciones pretenden buscar un equilibrio entre la necesidad de similitud y uniformidad, y la necesidad de flexibilidad, y considera el número de actores e instituciones involucrados. Las recomendaciones se agrupan en las siguientes tres categorías:

A. Fortalecimiento de las capacidades estadísticas y la eficacia del monitoreo Fortalecer la capacidad estadística a nivel local con énfasis en la recolección de datos, la calidad de la información obtenida de la encuesta, la evaluación estadística y el análisis. Cabe destacar que la actualización de los indicadores se logrará con la información que el Instituto Nacional de Estadística y Geografía (INEGI) publique en mayo del 2006, la cual representa el conteo de población y acceso a servicios aplicado en el año 2005.

Mejorar el uso de la información del monitoreo a través del fortalecimiento de las capacidades a fin de integrar los resultados de los análisis estadísticos a la formulación de políticas, la planificación, la asignación de recursos y el monitoreo posterior. 
Aplicar las experiencias importantes de monitoreo y usar metodologías que se concentren en un rango más amplio de variables, como el acceso de los pobres a los servicios, la gestión, la funcionalidad, las conductas y el uso de los servicios de agua, con el propósito de que el monitoreo se traduzca en acciones.

B. Coordinación, intercambio de información y cooperación

Fomentar la colaboración entre programas y proyectos a nivel local y utilizar los documentos de estrategias para la reducción de la pobreza como una de las plataformas donde se pueden integrar los ODM y mejorar la coordinación y la cooperación.

Desplegar esfuerzos constantes para uniformar las definiciones y expandir los indicadores acordados, con la participación de todas las partes interesadas del país a fin de garantizar la aplicabilidad a la situación nacional (Frausto et al., 2005). Ello facilitará el monitoreo de los ODM a nivel nacional y permitirá armonizar los esfuerzos.

C. Fortalecimiento del monitoreo global y del programa de monitoreo conjunto en particular

Brindar apoyo constante y mejorado a las plataformas internacionales a fin de que puedan uniformar los términos y las definiciones y garantizar la validez del monitoreo, particularmente para pasar de la mera evaluación del acceso a instalaciones mejoradas, a la evaluación del acceso sostenido a fuentes seguras de agua.

Incrementar la capacidad del equipo del Observatorio Urbano de manera que pueda aplicar algunas de las recomendaciones anteriores. Asimismo, reforzar las capacidades del Cuerpo Académico en su línea de generación del conocimiento sobre el aprovechamiento del recursos natural agua, con el fin de consolidar proyectos y la colaboración internacional.

\section{FUENTES CONSULTADAS}

Frausto, O. et al. (2005). "Sistema de información de indicadores ONU-Hábitat”. Ciudades. Análisis de la coyuntura, teoría e historia urbana, 17, (68), México. 
Acceso al agua potable

Indicador clave

de desarrollo humano

Guedán, M. (2005). Los objetivos del milenio. Tomarse la cooperación verdaderamente en serio. España: Dykinson.

INEGI (200I). XII Censo General de Población y Vivienda 2000. Sistema de Consulta de Información Censal 2000, Instituto Nacional de Estadística Geografía e Informática. Disco compacto.

Sedesol (2004). Sistema de consulta de indicadores del sistema urbano nacional (en línea). Disponible en: http://habitat2.sedesol.gob.mx/sedesol/ scisunweb/index.php [2005, mayo].

Unceta, K. (200I).“Perspectivas para el desarrollo humano en la era de la globalización” en Ibarra P. y Uncenta K. (coords.), Ensayos sobre el desarrollo humano. España: Icaria. 